\title{
On the Boundary Control of a Flexible Robot Arm
}

\author{
Ömer Morgül \\ Bilkent University, Dept. of Electrical and Electronics Engineering \\ 06533, Bilkent, Ankara, Turkey
}

\begin{abstract}
We consider a flexible robot arm modeled as a single flexible link clamped to a rigid body. We assume that the system performs only planar motion. For this system, we pose two control problems; namely, the orientation and stabilization of the system. We propose a class of controllers to solve these problems.
\end{abstract}

\section{Introduction}

In this paper, we study the motion of a rigid body with a flexible beam clamped to it at one end, the other end of the beam is free. We assume that the whole configuration performs planar motion. For this structure we pose two control problems, which we refer to as "orientation" and "stabilization" problems. We propose a class of control laws which solve these problems. Our control laws consist of a torque law applied to the rigid body and a dynamic boundary force control law applied to the free end of the flexible link. We prove that the proposed control laws solve the control problems alluded to above.

\section{Problem Statement}

We consider a system which consists of a flexible beam clamped to a rigid hub at one end, is free at the other end and the whole system performs planar motion. For a figure of this system, see [2]-[4]. Let $L$ be the length of the beam, $Q$ be the point where the beam is clamped to the rigid hub, $b$ be the distance between the center of mass of the rigid hub and $Q$. Let $u(x, t)$ be the displacement of the beam at $x$, and $t \geq 0$. The relevant equations of motion are (in linearized form):

$$
\begin{aligned}
& \rho u_{t t}+E I u_{x x x x}+\rho(b+x) \ddot{\theta}=0, u(0, t)=0, \\
& I_{R} \ddot{\theta}=E I\left(-b u_{x x x}(0, t)+u_{x x}(0, t)\right)+N(t), \\
& u_{x}(0, t)=u_{x x}(L, t)=0, E I u_{x x x}(L, t)=f(t),
\end{aligned}
$$

where $N(t)$ is the control torque applied to the rigid hub, $f(t)$ is the boundary control force applied to the free end of the beam. For details, see [2].
For the system given by (1)-(3) the following control problems are posed :

Problem 1 : (stabilization problem) Consider the system given by (1)-(3). Find appropriate control laws for $N(t)$ and $f(t)$ such that an appropriate norm of the solutions $u(x, t), u_{t}(x, t)$ and $\theta(t)$ of (1)-(3) decay to 0 as $t \rightarrow \infty$.

Problem 2: (orientation problem) Consider the system given by (1)-(3). Let an angle $\theta_{0} \in[0,2 \pi)$ be given . Find appropriate control laws for $N(t)$ and $f(t)$ such that the stability problem is solved, moreover we have $\lim _{t \rightarrow \infty} \theta(t)=\theta_{0}$, where the angle $\theta_{0}$ is the orientation angle.

To generate the boundary control force $f(t)$ we propose the following class of controllers :

$$
\begin{gathered}
\dot{w}=A w+b u_{t}(L, t) \dot{z}_{2}=-\omega_{1} z_{1}+u_{t}(L, t) \\
\dot{z}_{1}=\omega_{1} z_{2}, f(t)=c^{T} w+d u_{t}(L, t)+k u(L, t)+k_{2} z_{2}
\end{gathered}
$$

where $w \in \mathbf{R}^{n}$ is the actuator state, $A \in \mathbf{R}^{n \times n}$ is a constant matrix, $b, c \in \mathbf{R}^{n}$ are constant column vectors, $d, k, k_{2}$ are a constant real numbers, the superscript $T$ stands for transpose. If we take the Laplace transform, then the controller transfer function $g(s)$ between its input $u_{t}(1, t)$ and output $f(t)$ may be found as

$$
g(s)=g_{1}(s)+\frac{k}{s}+\frac{k_{2} s}{s^{2}+\omega_{1}^{2}}
$$

where $g_{1}(s)=c^{T}(s I-A)^{-1} b+d$. We assume the following throughout the paper :

Assumption 1 : $A$ is Hurwitz stable and the triple $(A, b, c)$ is minimal.

Assumption 2 : $d \geq 0$; moreover there exists a constant $\gamma$, such that $d \geq \gamma \geq 0$, and that the following holds :

$$
d+\operatorname{Re}\left\{c^{T}(j \omega I-A)^{-1} b\right\}>\gamma, \quad, \quad \omega \in \mathbf{R} .
$$

Moreover, for $d>0$, we require $\gamma>0$ as well.

To generate the control torque $N(t)$, we propose the following control law :

$$
N(t)=(b+L) f(t)-k_{p} \dot{\theta}-k_{i}\left(\theta-\theta_{0}\right),
$$

where $k_{p}, k_{i}$ are constant real numbers. 


\section{Stability Results}

\section{i: Stabilization Problem}

For the sake of brevity, in the sequel we call the system given by (1)-(3), (4)-(5), (8) with $k_{i}=0$ as system $\mathcal{S}_{1}$. To analyze the system $\mathcal{S}_{1}$, we first define the function

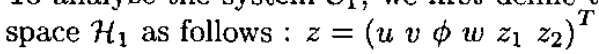

$$
\mathcal{H}_{1}=\left\{z \mid u \in \mathbf{H}_{0}^{2}, v \in \mathbf{L}^{2}, \phi, z_{1}, z_{2} \in \mathbf{R}, w \in \mathbf{R}^{n}\right\}
$$

for the definition of various spaces, see e.g. [2], [3].

The equations of the system $\mathcal{S}_{1}$ can be written in the following abstract form :

$$
\dot{z}=A_{1} z \quad, \quad z(0) \in \mathcal{H}_{1}
$$

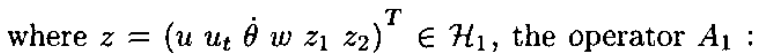
$\mathcal{H}_{1} \rightarrow \mathcal{H}_{1}$ is a linear unbounded operator.

Theorem 1 : Consider the system given by (10). Let $k_{p}>0, d \geq 0, k \geq 0, k_{2} \geq 0$, and let the assumptions 1-2 hold, (Note that $k_{i}=0$ ). Then,

i : The operator $A_{1}$ generates a $C_{0}$-semigroup of contractions $T_{1}(t)$ on $\mathcal{H}_{1}$; moreover, if $z(0) \in D\left(A_{1}\right)$, then $z(t)=T_{1}(t) z(0), t \geq 0$, is the unique classical solution of (10) and $z(t) \in D\left(A_{1}\right)$ for $t \geq 0$, (for the terminology on semigroup theory, the reader is referred to e.g. [1]).

ii : If $k_{2}=0$, stabilization problem is solved in asymptotical sense in general, and is solved in exponential sense when $d>0$.

iii : If $k_{2}>0$, the stabilization problem is solved in asymptotical sense if $\tau=\sqrt{\omega_{1}}$ is not a root of the following equation :

$$
\cosh \tau \sin \tau-\sinh \tau \cos \tau=0 .
$$

Proof : Proof of this fact requires some length and is omitted here due to space limitations.

\section{ii : Orientation Problem}

Let $\theta_{e}$ be the error angle defined as $\theta_{e}=\theta-\theta_{0}$ Since $\theta_{0}$ is a constant, it follows that $\dot{\theta}=\dot{\theta}_{e}$.

For the sake of brevity, in the sequel we call the system given by (1)-(3), (4)-(5), (8) with $k_{i}>0$ as system $\mathcal{S}_{2}$. The equations of the system $\mathcal{S}_{2}$ can be written in the following abstract form :

$$
\dot{z}=A_{2} z \quad, \quad z(0) \in \mathcal{H}_{2},
$$

where $\mathcal{H}_{2}=\mathcal{H}_{1} \times \mathbf{R}, z=\left(\begin{array}{lllll}u & \theta_{e} \dot{\theta}_{e} w z_{1} & z_{2}\end{array}\right)^{T} \in \mathcal{H}_{2}$, the operator $A_{2}: \mathcal{H}_{2} \rightarrow \mathcal{H}_{2}$ is a linear unbounded operator.
Theorem 2 : Consider the system given by (12). Let $k_{p}>0, k_{i}>0, d \geq 0, k \geq 0, k_{2} \geq 0$, and let the assumptions 1-2 hold, Then,

i : The operator $A_{2}$ generates a $C_{0}$-semigroup of contractions $T_{2}(t)$ on $\mathcal{H}_{2} ;$ moreover, if $z(0) \in D\left(A_{2}\right)$, then $z(t)=T_{2}(t) z(0), t \geq 0$, is the unique classical solution of (12) and $z(t) \in D\left(A_{2}\right)$ for $t \geq 0$, (for the terminology on semigroup theory, the reader is referred to e.g. [1]).

ii : If $k_{2}=0$, stabilization problem is solved in asymptotical sense in general, and is solved in exponential sense when $d>0$.

iii : If $k_{2}>0$, the stabilization problem is solved in asymptotical sense if $\tau=\sqrt{\omega_{1}}$ is not a root of (11).

Proof : Proof of this Theorem is similar to that of Theorem 1, requires some length and hence is omitted here due to space limitations.

\section{Conclusion}

In this paper we studied the planar motion of a flexible structure which consists of a flexible beam clamped to a rigid hub. Such a structure may model a robot arm with a single flexible link, or a communication satelite with a flexible antenna. We posed an orientation and a stabilization problem for this configuration. To control this structure we assumed that a control torque is applied to the rigid hub, and a boundary control force is applied to the free end of the flexible beam. To solve these problems we proposed a set of controllers. We then proved that the proposed controllers solve the stabilization and orientation problems in asymptotical sense in general, and in exponential sense for some cases.

\section{References}

[1] Z.H.Luo, B.Z.Guo, and Ö. Morgül, Stability and Stabilization of Infinite Dimensional Systems with Applications, Springer-Verlag, series in Communications and Contr. Eng., London, 1999.

[2] Ö. Morgül, "Orientation and stabilization of a flexible beam attached to a rigid body : planar motion," IEEE Trans. on Auto. Control, vol. 36, pp. 953$963,1991$.

[3] Ö. Morgül, "Control and stabilization of a flexible beam attached to a rigid body," International $J$. of Control, Vol. 51, No. 1, pp.11-31, 1990.

[4] Ö. Morgül, "Dynamic boundary control of a Euler-Bernoulli beam," IEEE Trans. on Auto. Control, vol. 37, No. 5, pp. 639-642, 1992. 